\title{
Bending Angle Effects on the Multi-Domain in-Plane-Switching Liquid Crystal Displays
}

\author{
Ruibo Lu, Shin-Tson Wu, Fellow, IEEE, Zhibing Ge, Qi Hong, and Thomas X. Wu, Senior Member, IEEE
}

\begin{abstract}
The electro-optic properties of a multi-domain in-plane switching liquid crystal display (IPS LCD) are investigated through a three-dimensional (3-D) simulator, which combines the finite element and finite difference methods for fast solutions. The bending angle between the chevron-shaped electrodes makes an important contribution to the operating voltage and response time, and suppresses the color shift. Using optimized uniaxial compensation films, the viewing cone of the multi-domain IPS LCD is widened to $\pm 80^{\circ}$ at 100:1 contrast ratio. These properties are especially desirable for LCD TV and computer monitor applications.
\end{abstract}

Index Terms-Liquid crystal displays (LCDs), in-plane switching (IPS), multi-domain, response time, wide viewing angle, color shift, phase compensation films.

\section{INTRODUCTION}

$\mathbf{L}$ IQUID CRYSTAL displays (LCDs) have been widely used for graphics, data, and TVs. Fast response time, high contrast ratio, wide viewing angle, and weak color shift are the major technical thrusts for large screen LCD TV and computer monitor applications [1]. To widen viewing angle, multi-domain vertical alignment (MVA) [2], [3], patterned vertical alignment (PVA) [4], [5], advanced super view (ASV) [6], [7], and in-plane switching (IPS) modes [8]-[10] have been developed. IPS mode inherently exhibits a wide view angle due to the in-plane reorientation of the LC directors. However, the conventional IPS LCD which uses stripe electrodes exhibits an apparent color shift at some specific viewing directions. In a super-IPS mode the zigzag electrode structure helps to reduce color shift [11], [12], but the intrinsic response time is still an issue. In addition, the viewing angle is still inadequate for TV applications. Compensation films are needed to reduce the light leakage of crossed polarizers at oblique angles.

Several methods for improving LC response time have been proposed [1]. Besides low viscosity LC materials, the most commonly adopted approach is to use overdrive and undershoot voltages [13]. For improving the IPS response time, Xiang et al. proposed a double-side fringe-field switching method [14], [15], and Sun et al. studied the optimal rubbing angle effect [16]. To widen viewing angle, phase compensation film is a favorable approach. Chen et al. proposed to use uniaxial films to improve the

Manuscript received June 6, 2005; revised July 24, 2005. This work is supported by Toppoly Optoelectronics Corporation (Taiwan), Taiwan, R.O.C.

R. Lu and S.-T. Wu are with the College of Optics and Photonics, University of Central Florida, Orlando, FL 32816 USA (e-mail: rlu@creol.ucf.edu; swu@creol.ucf.edu).

Z. Ge, Q. Hong, and T. X. Wu are with the Department of Electrical and Computer Engineering, University of Central Florida, Orlando, FL 32816 USA (e-mail: zge@ mail.ucf.edu; tomwu@ mail.ucf.edu; qhong@ mail.ucf.edu).

Digital Object Identifier 10.1109/JDT.2005.858941 viewing angle [17], while Saitoh et al. extended the 50:1 contrast ratio contour to the entire viewing cone by using biaxial compensation films [18].

In this paper, we analyzed the bending-angle effect of the chevron-shaped electrodes in the multi-domain (MD) IPS-LCD. We developed a reliable three-dimensional (3-D) dynamic model which combines the finite element method (FEM) and finite difference method (FDM) for simulating the LCD performances. The calculation time is reasonably fast [19]. The electro-optic properties of the MD IPS mode under different bending angles were characterized by the voltage-dependent transmittance (VT) curve, time-dependent transmittance curve, angular-dependent VT curve, color shift, and iso-contrast view angle contour. The viewing angle is widened using the optimized uniaxial compensation films. We demonstrated a MD IPS-LCD with fast response time and enhanced view angle while eliminating the angular-dependent VT variation and color shift.

\section{NumericAl APPROACHES AND OPTICAL CALCUlations}

The model used here is based on the Gibbs free energy formulation [20]. It consists of the calculation of the electric and elastic potential distribution and the associated director distribution within the time-step procedure, where the director and potential distributions are modeled via FEM while FDM is used as the base for the time step process [21], [22].

The LC director distributions are calculated by minimizing the total free energy, which is the summation of the electric free energy and the elastic free energy:

$$
f_{g}=f_{S}-f_{E}
$$

where $f_{g}, f_{S}$, and $f_{E}$ are the total free energy, elastic free energy, and electric free energy, respectively. Choosing the glass substrate parallel to the $(x, y)$ plane of a Cartesian coordinate system $(x, y, z)$, from Lagrange equation we find that the LC director profile is given by [23]

$$
\gamma_{1} \frac{d n_{i}}{d t}=-\left[f_{g}\right]_{n_{i}}+\lambda n_{i}
$$

where $n_{i}, \gamma_{1}, \lambda$, and $\left[f_{g}\right]_{\mathrm{ni}}$ are, respectively, the director vector in the $x, y, z$ direction, rotational viscosity, Lagrange multiplier for maintaining the unit length of the director, and free energy in the direction of the director vector. The total free energy in the direction of the director vector can be written as

$$
\begin{aligned}
{\left[f_{g}\right]_{n_{i}}=\frac{\partial f_{g}}{\partial n_{i}} } & -\frac{d}{d x}\left[\frac{\partial f_{g}}{\partial\left(d n_{i} / d x\right)}\right] \\
& -\frac{d}{d y}\left[\frac{\partial f_{g}}{\partial\left(d n_{i} / d y\right)}\right]-\frac{d}{d z}\left[\frac{\partial f_{g}}{\partial\left(d n_{i} / d z\right)}\right]
\end{aligned}
$$


Substituting (3) to (1), after simplification the separated form of the total free energy is related to the elastic free energy and the electric free energy as

$$
\left[f_{g}\right]_{n_{i}}=\left[f_{S}\right]_{n_{i}}-\left[f_{E}\right]_{n_{i}} .
$$

The elastic free energy $f_{S}$, is calculated from the Ossen-Frank free energy equation [20]

$$
\begin{aligned}
f_{S}=\frac{1}{2} K_{11}(\nabla \cdot \hat{n})^{2}+\frac{1}{2} K_{22}(\hat{n} & \left.\cdot \nabla \times \hat{n}+q_{0}\right) \\
+ & \frac{1}{2} K_{33}|(\hat{n} \times \nabla \times \hat{n})|^{2}
\end{aligned}
$$

where $q_{o}$ is the inherent chiral term, $\hat{n}$ is the director vector, $K_{11}, K_{22}$ and $K_{33}$ are the splay, twist, and bend elastic constant, respectively.

The electric free energy, $f_{E}$, under the effect of a static electric field which is induced by a constant voltage $V$ is given by

$$
\begin{aligned}
f_{E} & =\frac{1}{2} \bar{D} \cdot \bar{E}=\frac{1}{2}[\underline{\underline{\varepsilon}} \cdot(-\nabla V) \cdot(-\nabla V)] \\
& =\frac{1}{2} \underline{\underline{\varepsilon}} \cdot \nabla V \cdot \nabla V
\end{aligned}
$$

where $\bar{D}, \bar{E}$, and $\underline{\underline{\varepsilon}}$ are the electric flux density, the electric field intensity, and the LC dielectric constant matrix, respectively. The potential distribution could be solved as a variational problem using FEM, where the LC device is discretized by using first-order rectangular element and the periodic boundary condition is applied in the $(x, y)$ plane. Strong anchoring is assumed to the LC directors and this Dirichlet boundary condition is also imposed on the resultant matrix equations for the electric potentials.

Substituting (5) and (6) back to (3), the temporal director distribution could be solved using FDM as:

$$
n_{j}=n_{i}-\frac{\Delta t}{\gamma_{1}}\left[f_{g}\right]_{n i}
$$

where $\Delta t$ is the time step of the iteration. Therefore, assuming a fixed initial director distribution, a variational potential distribution can be obtained within each time step using FEM. The solution for the director distribution is further performed using FDM by assuming the above variational potential distribution as a fixed one. These two procedures are repeated within each time step until the steady state director distribution is obtained by the two-step iterative calculations.

With the known steady-state director distributions, the optical properties of the LC device such as in the average pixel transmittance and its variation as a function of the applied voltage, time, and view angle could be evaluated using the extended Jones Matrix method [24], [25]. The LC layer is modeled as a stack of uniaxial homogeneous layers with the same structure as the previous model [26]. Here, we assume the reflections between interfaces are negligible. Therefore, the transmitted electric field is related to the incident electric field by [25]

$$
\begin{aligned}
{\left[\begin{array}{l}
E_{x} \\
E_{y}
\end{array}\right]_{N+1} } & =\mathbf{J}\left[\begin{array}{l}
E_{x} \\
E_{y}
\end{array}\right]_{1} \\
& =\mathbf{J}_{\mathrm{Ext}} \mathbf{J}_{N} \mathbf{J}_{N-1} \ldots \mathbf{J}_{2} \mathbf{J}_{1} \mathbf{J}_{\mathrm{Ent}}\left[\begin{array}{c}
E_{x} \\
E_{y}
\end{array}\right]_{1}
\end{aligned}
$$

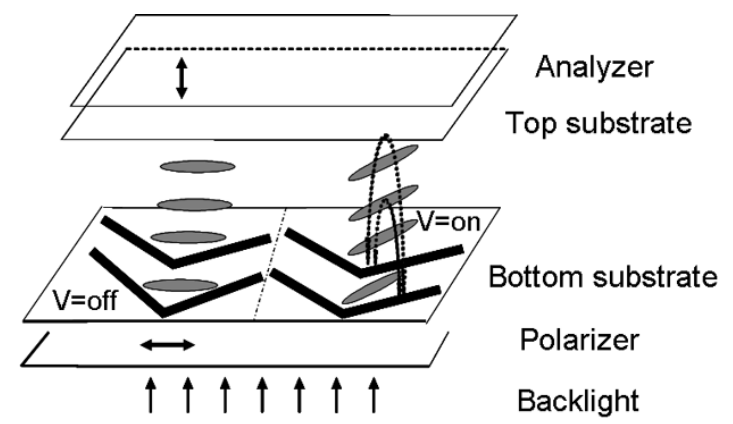

(a)



(b)

Fig. 1. (a) Typical structure of MD IPS mode LCD and (b) its working principles.

where $J_{\text {Ext }}$ and $\mathbf{J}_{\text {Ent }}$ are the correction matrix considering the transmission losses in the air-LCD interface, which are given by

$$
\begin{aligned}
\mathbf{J}_{\mathrm{Ent}} & =\left[\begin{array}{lc}
\frac{2 \cos \theta_{p}}{\cos \theta_{p}+n_{p} \cos \theta_{k}} & 0 \\
0 & \frac{2 \cos \theta_{k}}{\cos \theta_{k}+n_{p} \cos \theta_{p}}
\end{array}\right], \\
\mathbf{J}_{\mathrm{Ext}} & =\left[\begin{array}{lc}
\frac{2 n_{p} \cos \theta_{k}}{\cos \theta_{p}+n_{p} \cos \theta_{k}} & 0 \\
0 & \frac{2 n_{p} \cos \theta_{p}}{\cos \theta_{k}+n_{p} \cos \theta_{p}}
\end{array}\right]
\end{aligned}
$$

Correspondingly, the overall optical transmittance is represented as

$$
t_{\mathrm{op}}=\frac{\left|E_{x, N+1}\right|^{2}+\cos ^{2}\left(\theta_{p}\right)\left|E_{y, N+1}\right|^{2}}{\left|E_{x, 1}\right|^{2}+\cos ^{2}\left(\theta_{p}\right)\left|E_{y, 1}\right|^{2}}
$$

where $n_{p}$ is the index of refraction of the polarizer, and $\theta_{p}$ is given by

$$
\theta_{p}=\sin ^{-1}\left(\sin \left(\theta_{k}\right) / \operatorname{Re}\left(\left(n_{e, p}+n_{o, p}\right) / 2\right)\right)
$$

in which $n_{e, p}$ and $n_{o, p}$ are the two refractive indices of the polarizer, and $\theta_{k}$ is the azimuthal angle of the incident wavevector $\mathrm{k}$.

\section{Device Structure ANd Working Principle}

Fig. 1 show the device structure and its working principle of the simulated multidomain IPS LCD, where both the positive and negative dielectric anisotropy LC materials can be used. The rubbing direction is preferred to be along the vertical direction in Fig. 1(a) and the LC directors are aligned homogeneously on the glass substrates. The chevron-shaped electrode is bent and has a bending angle, $\alpha$, which is set as the angle between the chevron arm extension direction and the vertical direction. A 


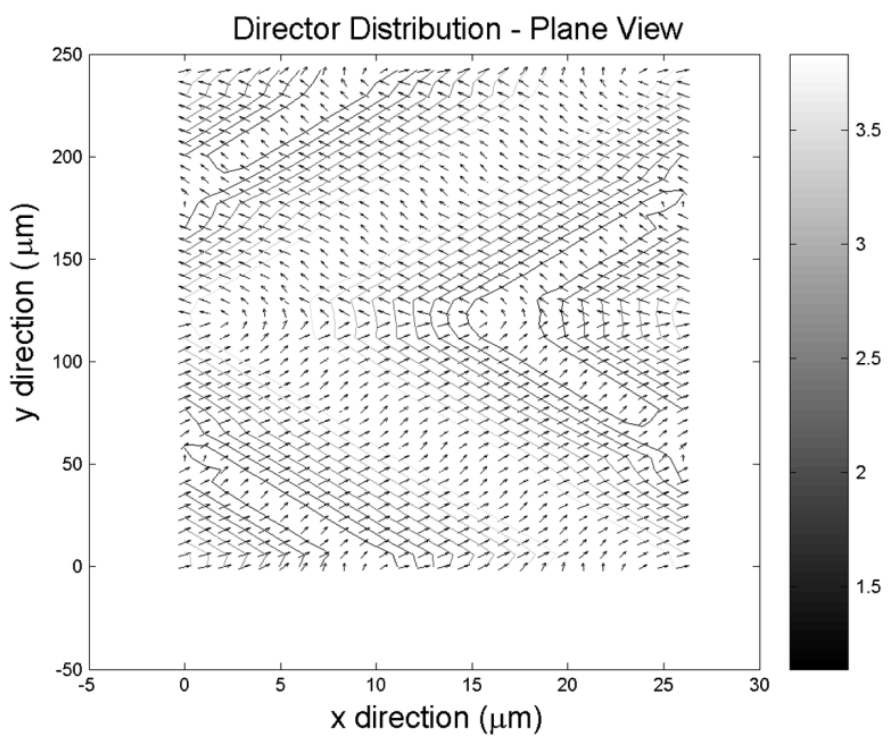

Fig. 2. Simulated LC directors distribution which is the center layer of MD IPS mode at the bending angle of $30^{\circ}$ with the applied voltage of $6.0 \mathrm{~V}_{\mathrm{rms}}$.

serial of chevron-shaped electrodes are alternatively arranged to form the inter-digital electrodes on the same substrate as the common electrode and the pixel electrode, respectively, which are connected to the thin-film transistors (TFTs) in the practical LCD devices.

The principal axis of the linear polarizer near the bottom substrate is parallel to the LC alignment (i.e., rubbing) direction. When there is no voltage applied, the incident light is completely blocked by the crossed polarizers, which results in a normally black state. When the applied voltage exceeds the threshold, the transversal electric fields are created and the electric field lines are in the parabolic form in the whole display area. Correspondingly, the LC directors with positive (or negative) dielectric anisotropy between the electrodes would be reoriented along (or perpendicular) the electric field direction. The LC directors above two chevron arm regions are twisted into different directions so that the multi-domain LC configuration is generated and light transmits through the crossed polarizers.

As an example, we simulate a chevron-shaped multi-domain IPS LCD using a Merck positive LC material MLC 6692. Its physical properties are summarized as follows: $\gamma_{1}=0.1$ Pas, $\Delta \varepsilon=10.3, \mathrm{~K}_{11}=9.6 \mathrm{pN}, \mathrm{K}_{22}=6.1 \mathrm{pN}, \mathrm{K}_{33}=14.1 \mathrm{pN}$, and $\Delta \mathrm{n}=0.085$ at $\lambda=550 \mathrm{~nm}$. The cell gap is $\mathrm{d}=4 \mu \mathrm{m}$, the width of the chevron-shaped electrode is $w=3 \mu \mathrm{m}$, the electrode gap is $g=8 \mu \mathrm{m}$, and the chevron arm length is $\ell=128 \mu \mathrm{m}$. The bending angle of the chevron-shaped electrodes, $\alpha$, is varied from $10^{\circ}$ to $45^{\circ}$. The employed polarizers are Nitto Denko HEG1425 types with a thickness of $210 \mu \mathrm{m}$. The maximum transmittance of the polarizer/analyzer pair is $35 \%$.

\section{RESULTS AND DisCUSSION}

\section{A. LC Directors Distribution and Light Transmittance}

Fig. 2, plots the LC director distribution in the central layer of a multi-domain IPS LC cell at $\alpha=30^{\circ}$ and $V=6.0 \mathrm{~V}_{\mathrm{rms}}$. It is clearly shown that the LC directors are twisted into different directions in-between the neighboring electrodes; especially, the
LC directors on the two chevron arm regions are switched into complementary directions. Therefore, the chevron-shaped electrodes help to form four-domain structure.

Fig. 3 plots the corresponding 3-D light transmittance contour of the multidomain IPS LCD through the crossed linear polarizers. It can be found that the regions in-between the neighboring electrodes predominantly contribute to the transmittance because the LC directors are uniformly twisted in these regions. The LC directors above the electrodes are only partially switched or even unmoved, resulting in a low transmittance. These regions appear dark under crossed polarizers and form the so-called dead zones or domain-walls as shown in Fig. 2. The appearance of the dead zones reduces the overall light transmittance. To minimize dead zones, we could narrow the electrode gap and make the full use of the fringe-field switching (FFS) effect [27], or use the finger-on-plane (FOP) structure [28].

\section{B. Voltage-Dependent Transmittance (VT)}

The voltage-dependent transmittance (VT) curves of the conventional IPS mode and the multidomain IPS mode with $\alpha=$ $10^{\circ}$ bending angle are depicted in Fig. 4(a) and (b), respectively. The conventional IPS mode has a similar device configuration to the MD IPS mode but with the stripe electrodes. The rubbing angle is defined as the angle between the LC orientation direction and the electrode longitudinal direction. The three primary colors used for simulations are $R=650, G=550$, and $B=$ $450 \mathrm{~nm}$. In Fig. 4(a), as the applied voltage exceeds $1.2 \mathrm{~V}_{\mathrm{rms}}$, the transmittance begins to increase and reaches its respective maximum at $4.75 \mathrm{~V}_{\mathrm{rms}}$ for the different wavelengths. The maximum transmittance is $29 \%$ for the green wavelength. For the multidomain IPS mode shown in Fig. 4(b), the VT curves are similar to those of the conventional IPS mode, except for a slightly higher on-state voltage $\left(5 \mathrm{~V}_{\mathrm{rms}}\right)$ and a slightly lower maximum transmittance $(27.8 \%)$. The $\sim 1 \%$ decrease in maximum transmittance is due to the multidomain formation [29], [30], which lowers the effective phase retardation.

Fig. 5 plots the VT curves of the MD IPS cell at $\lambda=550$ $\mathrm{nm}$ and different bending angles. As the bendnig angle increases, the maximum transmittance gradually decreases and the on-state voltage increases. For instance, the maximum transmittance occurs at $5 \mathrm{~V}_{\mathrm{rms}}$ for $\alpha=10^{\circ}$, but increases to 7 $\mathrm{V}_{\mathrm{rms}}$ as the bending angle increases to $\alpha=40^{\circ}$. The higher on-state voltage is due to the required effective projected electric field to switch the LC directors when the bending angle of the chevron electrode increases. In the meantime, the threshold voltage is lowered from 1.25 to $0.75 \mathrm{~V}_{\mathrm{rms}}$ as the bending angle is increased from at $\alpha=10^{\circ}$ to $40^{\circ}$.

\section{Time-Dependent Transmittance}

Fig. 6 plots the time-dependent transmittance under the pulsed squarewave voltages, which correspond to the maximum transmittance shown in Fig. 5. As the bending angle increases, the rise time becomes shorter, but its maximum transmittance is lower. The decay time remains unchanged. The faster response time for a larger tilt angle is because the higher applied voltage and lower threshold. Thus, the effective 


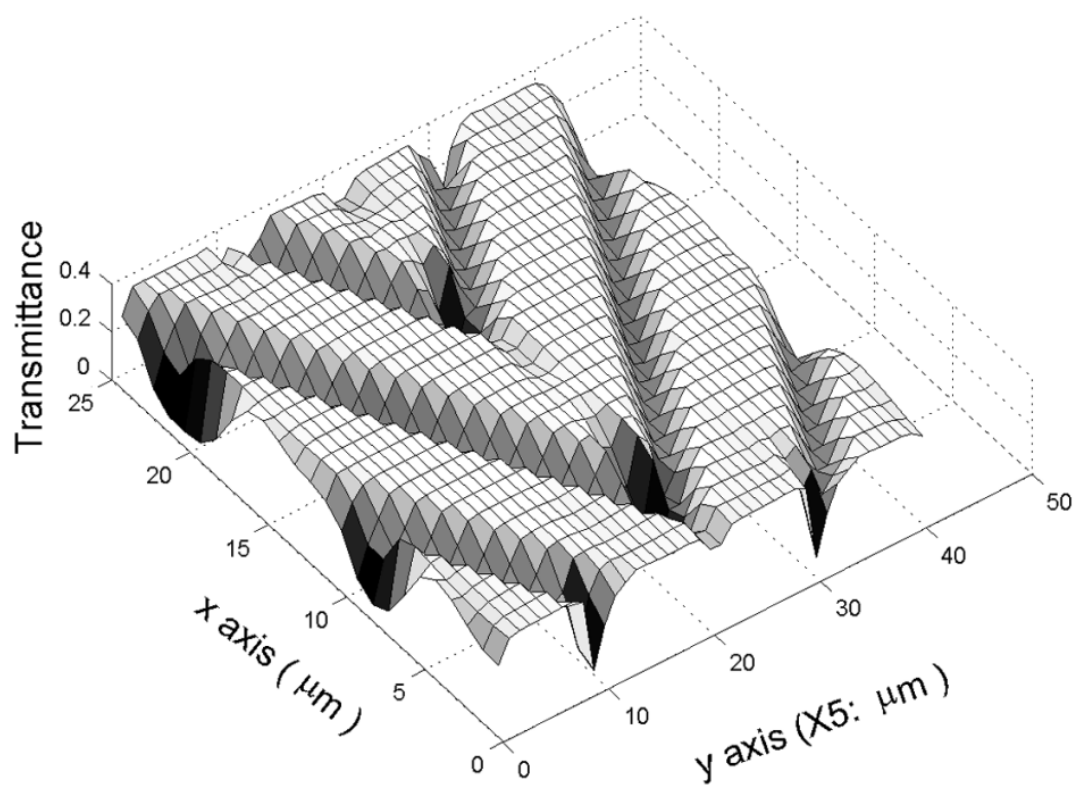

Fig. 3. The 3-D light transmittance contribution of the MD IPS mode LCD through the crossed linear polarizers, where the bending angle is $30^{\circ}$ and the applied voltage $6.0 \mathrm{~V}_{\mathrm{rms}}$.
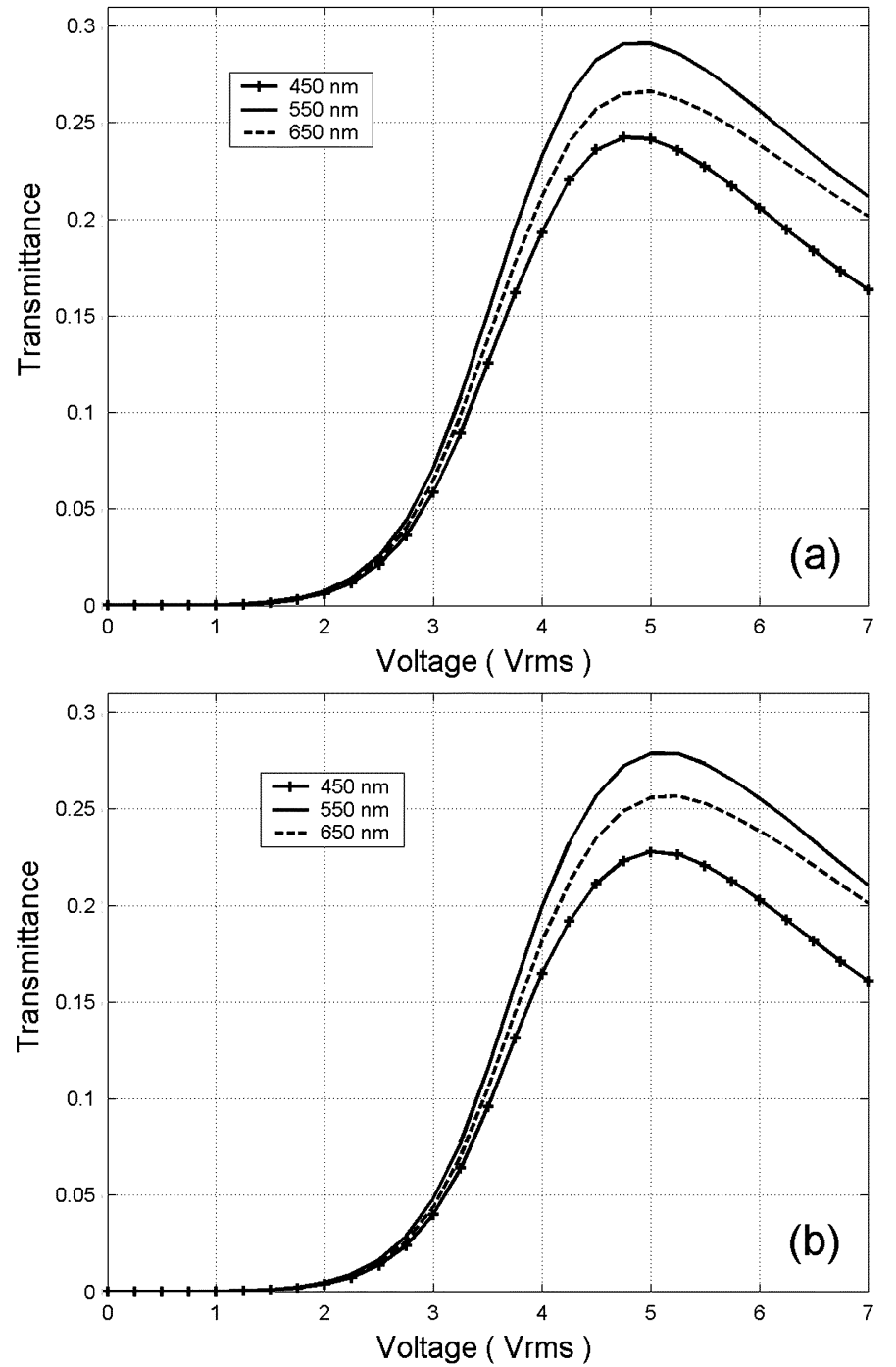

Fig. 4. Voltage-dependent light transmittances of the different IPS modes at the bending angle (or the rubbing angle) of $10^{\circ}$. (a) The conventional IPS mode. (b) The MD IPS mode. switching ratio $\left(V_{\mathrm{on}} / V_{\mathrm{th}}\right)$ is higher. A higher switching ratio leads to a faster rise time [1].

Fig. 7 shows the calculated response times based on Fig. 6, where the rise time is defined as the transmittance changes from $10 \%$ to $90 \%$ while the decay time is calculated from $90 \%$ to $10 \%$. As the bending angle increases the rise time decreases rapidly. For example, when the bending angle is increased from $10^{\circ}$ (this is also the common case for super-IPS LCD) to $20^{\circ}$ and $30^{\circ}$, the rise time is reduced from 42 to $22 \mathrm{~ms}$, and $18 \mathrm{~ms}$, respectively. However, the tradeoffs are lower maximum transmittance and higher operating voltage. The optimal electrode bending angle seems to occur at $\alpha \sim 20-35^{\circ}$.

Sun et al. have investigated the rubbing angle effect on a reflective IPS LCD [15]. This analysis can be extended to our device structure. Since our MD IPS LCD consists of a series of chevronshaped electrodes, it can be regarded as two stripe-shaped IPS cells with the respective initial tilt angle, $\alpha$ and $-\alpha$, that are driven by the electric field simultaneously. In addition, since the length of each chevron arm is much larger than the separation distance between the neighboring electrodes, the LC dynamic director rotation in-between the neighboring chevron arm regions can be simplified as the following Erickson-Leslie equation without the consideration of the backflow and inertial effects [10]:

$$
\gamma_{1} \frac{\partial \phi}{\partial t}=k_{2} \frac{\partial^{2} \phi}{\partial t^{2}}+\varepsilon_{0}|\Delta \varepsilon| E^{2} \sin \phi \cdot \cos \phi
$$

where $\gamma_{1}$ is LC rotational viscosity, $k_{2}$ is the twist elastic constant, $\Delta \varepsilon$ is the dielectric anisotropy, $E$ is the applied electric field strength, and $\phi$ is the LC rotation angle.

Correspondingly, the rise time and decay time can be estimated from the following expressions:

$$
\tau_{\text {rise }}=\frac{\gamma_{1}}{\varepsilon_{0}|\Delta \varepsilon| E^{2}\left[\sin (2 \alpha) \frac{\cos (2 \bar{x})}{2 \bar{x}}+\cos (2 \alpha) \frac{\sin (2 \bar{x})}{2 \bar{x}}\right]-\frac{\pi^{2}}{d^{2}} k_{2}}
$$

$$
\tau_{\text {decay }}=\frac{\gamma_{1} d^{2}}{\pi^{2} k_{2}}
$$




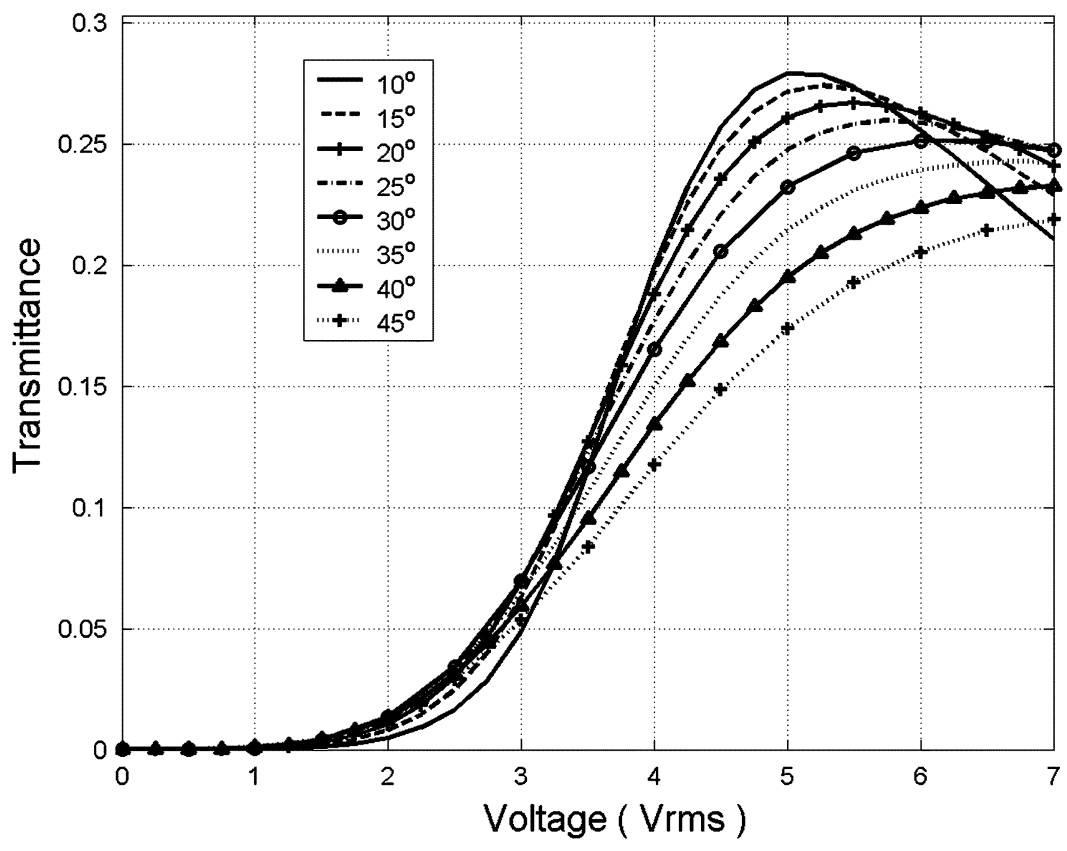

Fig. 5. Voltage-dependent transmittance of the MD IPS mode with the different bending angles at the wavelength of $550 \mathrm{~nm}$.



Fig. 6. Time-dependent transmittance of the MD IPS mode under the pulsed squarewave voltages at the different maximum transmittance voltage points.

where $d$ is the LC cell gap, and $\bar{x}$ is defined as $\bar{x}=\int_{0}^{(d / 2)} x \cdot d z$ that integrates the rotation angle of every LC layer along z-axis.

The above two equations reveal that the free relaxation time of an IPS mode mainly depends on the cell gap $d$, LC rotational viscosity $\gamma_{1}$, and the twist elastic constant $k_{2}$, while the rise time is also affected by the bending angle of the electrodes and the applied voltage. In a real MD IPS LCD, the situation is actually more complicated because it involves 3-D structures. From the above simplified analytical solutions, optimizing the bending angle could lead to response time improvement, which is consistent with our 3-D simulation results.

\section{Angular-Dependent VT Curves}

LC is a birefringent material, thus, the phase retardation in the voltage-off and-on states could depend on the viewing direction, especially in the large oblique angles. This phase difference leads to the undesirable angular-dependent VT curve variations and color shift.

In Fig. 8, Snell's law correlates the incident angle $\theta_{i}$ in the air to the exit angle $\theta$ in the $\mathrm{LC}$ medium as

$$
n_{\text {air }} \sin \theta_{i}=n \sin \theta .
$$




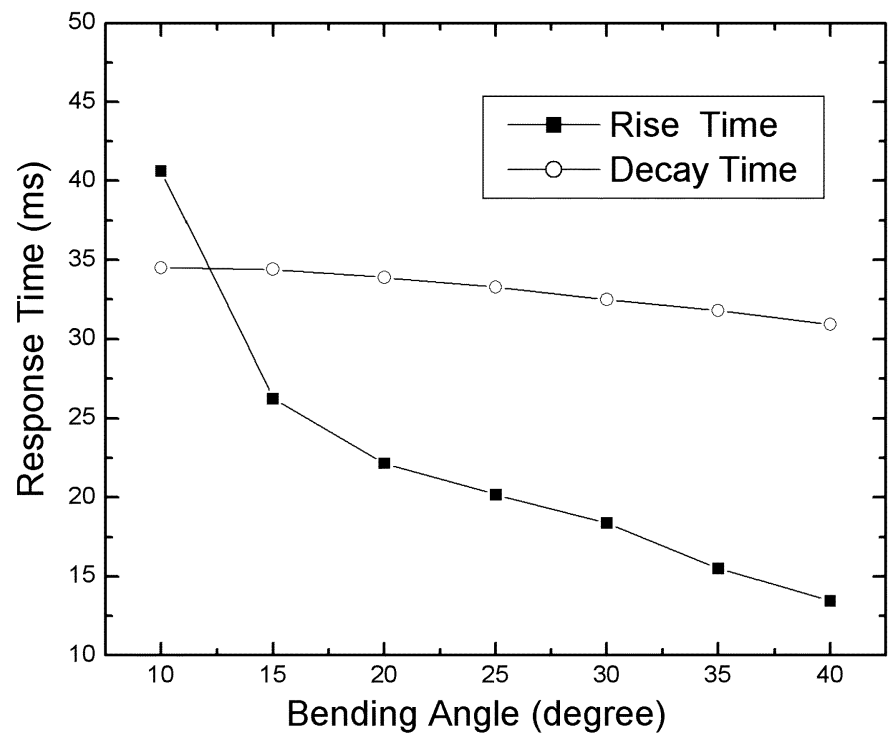

Fig. 7. Calculated response time of the MD IPS mode at the different tilt angles.

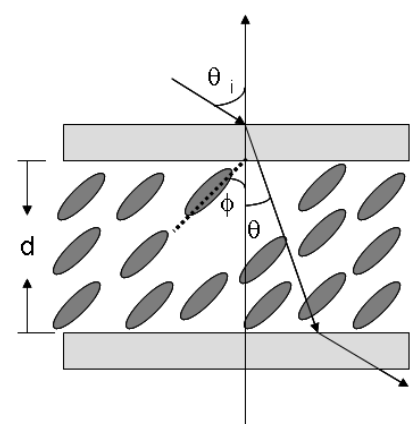

Fig. 8. Light path of a uniformly tilted liquid crystal cell at an oblique angle.

The angular dependent phase retardation of the LC medium at a given wavelength $\lambda$ is expressed as [31]

where

$$
\delta(\theta, \varphi, \phi, V, \lambda)=2 \pi(d \cdot \Delta n)_{\mathrm{eff}} / \lambda
$$

$$
\begin{aligned}
(d \cdot & \Delta n)_{\mathrm{eff}} \\
= & \frac{d}{\cos \theta}\left\{\frac{n_{e} n_{o}}{\sqrt{n_{o}^{2} \sin ^{2}(\theta \pm \phi)+n_{e}^{2} \cos ^{2}(\theta \pm \phi)}}-n_{o}\right\} .
\end{aligned}
$$

In (15) and (17), $n_{o}$ and $n_{e}$ represent the ordinary and extraordinary refractive indices, $\Delta n=n_{e}-n_{o}$ the birefringence, $n=\left(n_{e}+2 n_{o}\right) / 3$ the average refractive index of the LC material, $d$ is the LC cell gap, $\varphi$ is the angle between the effective optical axis of the LC directors and the transmission axis of the polarizer, and $\phi$ is the LC tilt angle. The \pm signs in (17) represent the light coming from left and right sides, respectively.

Correspondingly, the normalized light transmittance (T) through the LC medium under the crossed linear polarizers has the following form [32]:

$$
T=\sin ^{2}(2 \varphi) \sin ^{2}(\delta / 2)=\sin ^{2}(2 \varphi) \sin ^{2}\left[\pi \cdot(d \cdot \Delta n)_{\mathrm{eff}} / \lambda\right] .
$$
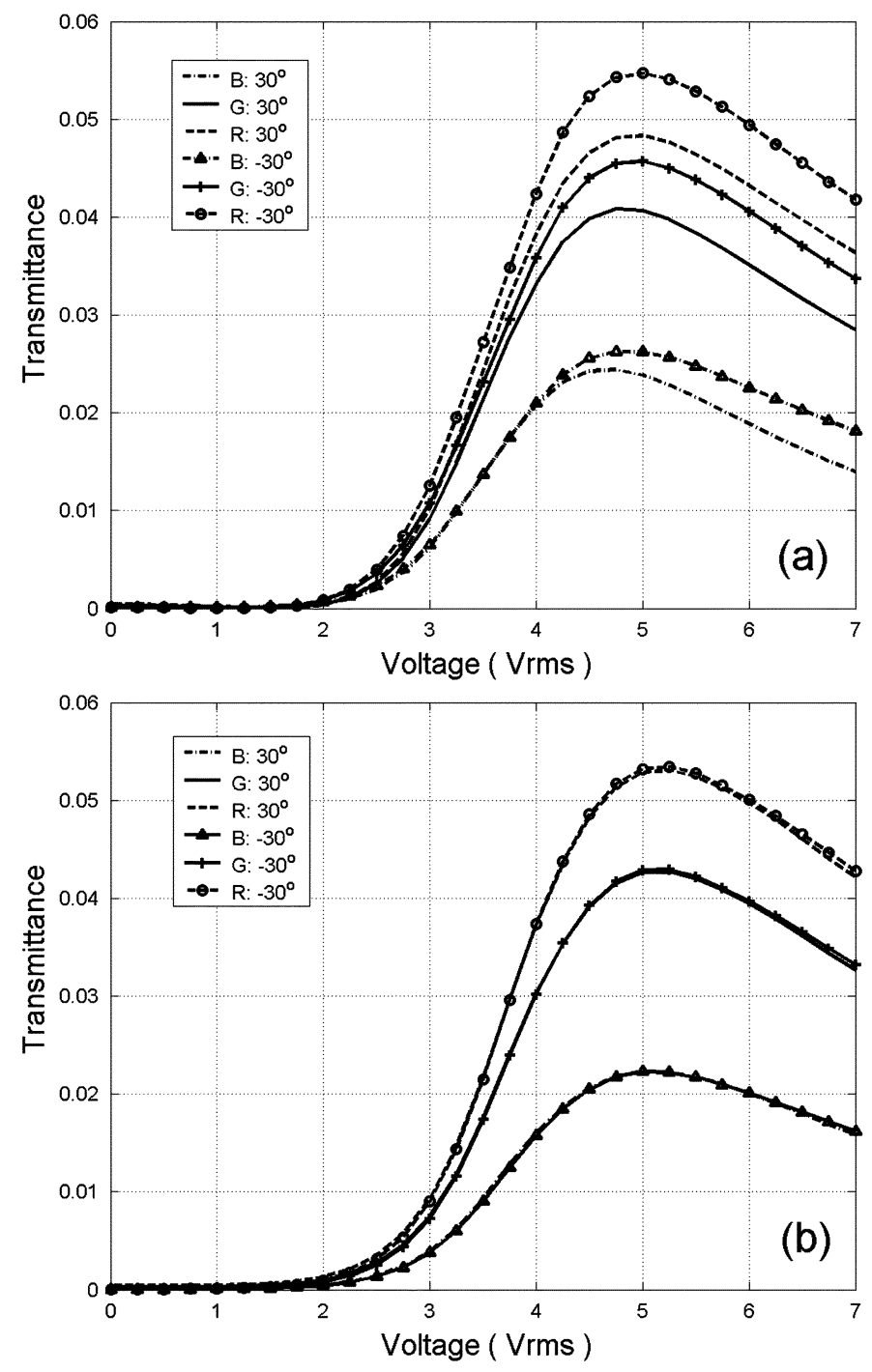

Fig. 9. Angular-dependent VT variation of the different IPS modes, where the azimuthal angle is set at $30^{\circ}$ and the incident light is from $\pm 30^{\circ}$, respectively. (a) The conventional IPS mode. (b) The MD IPS mode (bending angle at $30^{\circ}$ ).

Equation (18) indicates that the transmittance is closely related to the light incident angle, LC orientation angle, the incident wavelength, and the applied voltage. We quantify the angulardependent transmittance based on the VT curves at opposite incident angles and at RGB wavelengths.

Fig. 9(a) and (b) compares the simulated angular-dependent VT variation of a conventional IPS and the MD IPS LCDs, respectively. The azimuthal angle is set at $30^{\circ}$ and the incident angle at $\pm 30^{\circ}$. As shown in Fig. 9(a), the angular-dependent VT variation for the RGB colors is quite obvious for the conventional IPS mode when the applied voltage is higher than the respective threshold voltage. The largest variation usually occurs at the maximum transmittance. However, in the MD IPS mode the angular-dependent VT variation problem is completely eliminated, as shown in Fig. 9(b).

As shown in Fig. 2, when $V>V_{\text {th }}$ the LC directors in the MD IPS mode are twisted to different directions in-between the neighboring electrodes, and form different subdomains. Specially, the two subdomains residing in-between the two different electrode arm regions are twisted in the opposite directions, 

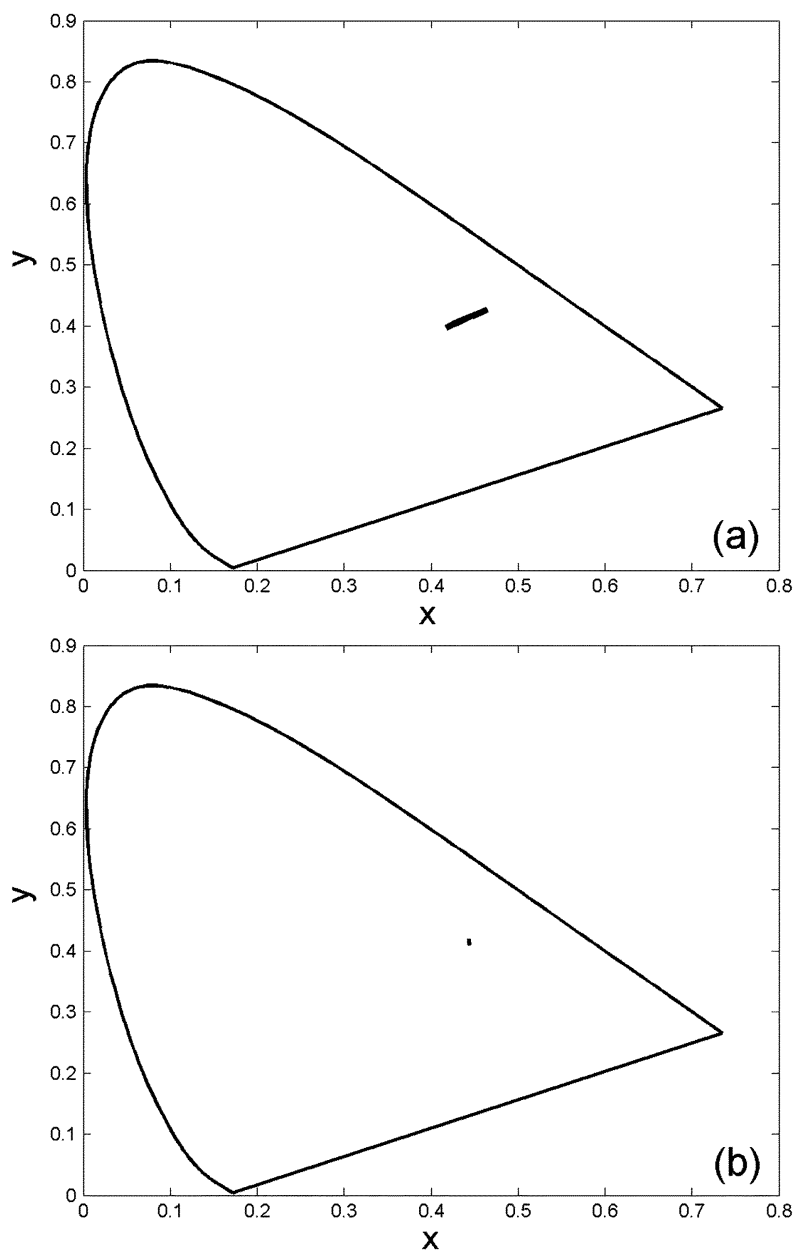

Fig. 10. The CIE 1931 chromaticity diagram of the different IPS modes at the incident angle of $50^{\circ}$ and scanned along the whole azimuthal range under the white light source. (a) The conventional IPS mode. (b) The MD IPS mode (bending angle at $30^{\circ}$ ).

which can compensate for the $(d \cdot \Delta n)_{\text {eff }}$ decrease in a subdomain by the $(d \cdot \Delta n)_{\text {eff }}$ increase from the opposite subdomain. It is helpful to eliminate the \pm sign effect from $\phi$ on the $(d \cdot \Delta n)_{\text {eff }}$ value when the incident light comes from the left or from the right side. Thus, the VT curve for a given wavelength is independent of the sign of $\phi$, i.e., the angular-dependent VT variation is unnoticeable.

\section{E. Bending Angle Effect on Color Shift}

From (18), the wavelength of the maximum transmittance shifts to a shorter wavelength when $(d \cdot \Delta n)_{\text {eff }}$ becomes smaller. That is to say, the white color shift occurs when $(d \cdot \Delta n)_{\mathrm{eff}}$ changes. Klausmann et al. [29] have investigated the color shift problem of a conventional IPS mode and found that for the rotated LC directors with an oblique angle parallel to the optical axis, the maximum value of $\sin ^{2}(\delta / 2)$ occurs at a shorter wavelength. That means the intended green color shifts toward blue range. In contrast, for the rotated LC directors with an oblique angle perpendicular to the optical axis, the maximum value of $\sin ^{2}(\delta / 2)$ happens at a longer wavelength, which means the appearance color shifts toward yellow range.
Fig. 10 compares the color shift (plotted in the CIE 1931 chromaticity diagram) of two different IPS modes at the maximum transmittance under different bending angles. The white light is incident from $50^{\circ}$ and scanned across the whole $360^{\circ}$ azimuthal range at $10^{\circ}$ scanning step. Fig. 10(a) plots the simulated color shifts of a conventional IPS mode at $\mathrm{V}=4.75 \mathrm{~V}_{\mathrm{rms}}$. A noticeable blue shift occurs at $30^{\circ}$ and $210^{\circ}$, and yellow shift at $130^{\circ}$ and $310^{\circ}$ azimuthal angles. On the contrary, in Fig. 10(b) no evident color shift is observed when the bending angle is as large as $30^{\circ}$ for the MD IPS mode operated at $V=6 \mathrm{~V}_{\mathrm{rms}}$. The multidomain structure not only widens the viewing angle but also suppresses the color shift.

The reason for the small color shift in MD IPS mode lies in that the LC directors are rotated into complementary directions in each sub-domain. The multidomain structure with the opposite twist directions in the neighboring two sub-domains compensates for the $(d \cdot \Delta n)_{\text {eff }}$ decrease at a longer wavelength in one sub-domain by the $(d \cdot \Delta n)_{\text {eff }}$ increase at a shorter wavelength in the opposite sub-domain. The light transmittance is kept constant at a set of incident angle on the whole azimuthal range when illuminated with a white light source. As a result, color shift in the chromaticity diagram is small.

\section{F. Viewing Angle Ability}

Fig. 11 plots the inherent iso-contrast contour bars of the abovementioned IPS modes. For the conventional IPS mode at $\mathrm{V}=4.75 \mathrm{~V}$ rms and $10^{\circ}$ rubbing angle, the iso-contrast bar is not symmetric and the viewing angle is relatively narrow $\left( \pm 65^{\circ}\right.$ at 10:1 contrast ratio). While in the in the MD IPS mode, as the tilt angle departs from zero the iso-contrast bar becomes symmetric along the horizontal/vertical direction and the view angle is wider. For the $30^{\circ}$ bending angle at $V=6 \mathrm{~V}_{\mathrm{rms}}$, its contrast ratio is higher than $500: 1$ at $\pm 25^{\circ}$ and the 10:1 iso-contrast view cone extends to $\pm 75^{\circ}$. In the horizontal and vertical directions, the viewing angles extend all the way to $\pm 90^{\circ}$ with contrast ratio higher than 100:1.

Chen [17] and Saitoh et al. [18] have demonstrated that the IPS viewing angle can be further improved using either a uniaxial or a biaxial compensation film. As seen from (17), if the incident angle $\theta_{i}$ is nonzero, then $(d \cdot \Delta n)_{\text {eff }} \neq 0$, i.e., the phase retardation from the LC medium exists and its value depends on the incident angle. This nonvanishing phase retardation would cause light leakage from the crossed polarizers. This off-axis light leakage is detrimental to the contrast ratio under the oblique angles which, in turn, affects the viewing angle performance. Therefore, a crucial issue for enhancing contrast ratio over a wide viewing range is to eliminate the off-axis light leakage and minimize the dark state transmittance.

To minimize the off-axis light leakage, it is necessary to select proper optical phase compensation films to cancel the residual LC phase retardation at any oblique angles:

$$
(d \cdot \Delta n)_{\mathrm{eff}}+(d \cdot \Delta n)_{\mathrm{film}} \approx 0 .
$$

Meanwhile, the dark state should remain as black as possible and insensitive to the viewing angle. These are the two basic criteria for implementing the phase compensation films. 

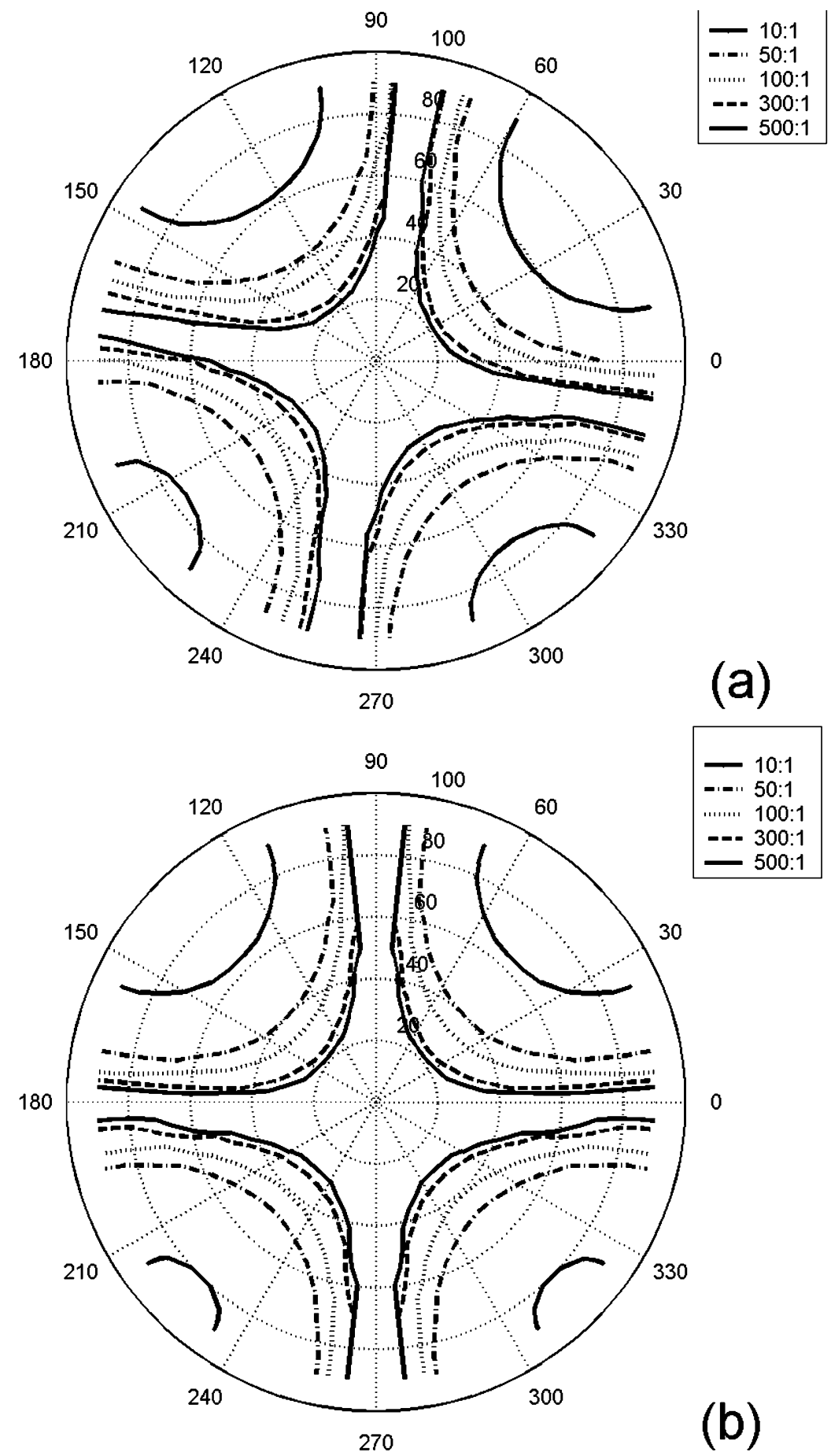

Fig. 11. Inherent iso-contrast contour bars of the different IPS modes. (a) The conventional IPS mode. (b) The MD IPS mode (bending angle at $30^{\circ}$ ).

Previously we have optimized the compensation films to widen the viewing angle of the conventional IPS LCD using uniaxial compensation films, where a positive $\mathrm{C}$-plate is placed after the LC cell and before the positive A-plate in-between the crossed polarizers [33]. Here, we use the similar method to optimize the compensation films to eliminate the off-axis light leakage of the MD IPS mode. The optimized positive C-plate has $d \Delta n=91.8 \mathrm{~nm}$, and is placed after the LC cell and before the optimized A-plate $(d \Delta n=155.4 \mathrm{~nm})$. Fig. 12(a) plots the iso-contrast bar of the MD IPS mode using the proposed compensation films, where the bending angle is $30^{\circ}$ and the applied voltage is $6 \mathrm{~V}_{\text {rms }}$. Simulation results show that the 500:1 contrast ratio is extended to over $\pm 50^{\circ}$ viewing cone and the $100: 1$ contrast ratio is wider than $\pm 80^{\circ}$ viewing range.

In reality, the film thickness may not be controlled precisely. Thus, we have to consider the manufacturing tolerance. In the extreme cases, we assume the $d \Delta n$ values of the A-plate and C-plate are simultaneously 5\% below or above their optimal values. Results are plotted in Fig. 12(b) and (c), respectively. In both cases, the viewing angle is slightly narrower, but their contrast ratio still maintains $\sim 100: 1$ at $\pm 70^{\circ}$ viewing cone. 


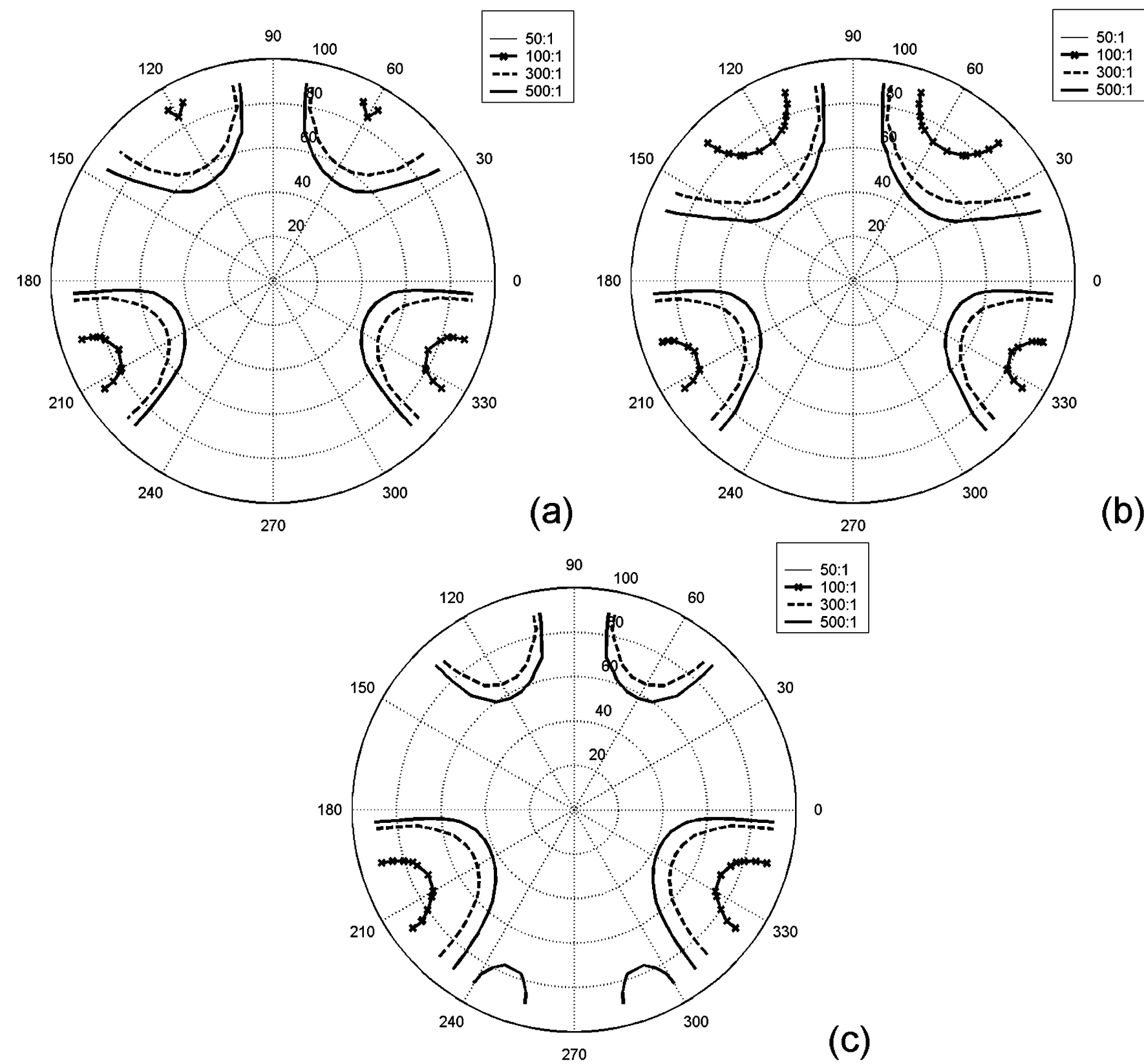

Fig. 12. (a) Isocontrast bar of the MD IPS mode using the optimized films, where the bending angle is $30^{\circ}$ and the applied voltage is $6 \mathrm{~V}_{\mathrm{rms}}$. The positive A-plate and C-plate films have the respective $d \cdot \Delta n$ value of 155.4 and $91.8 \mathrm{~nm}$. (b) $d \cdot \Delta n$ of the A-plate and C-plate are both $5 \%$ below the optimal values, and (c) $d \cdot \Delta n$ of the A-plate and C-plate are both $5 \%$ above the optimal values.

\section{CONCLUSION}

The electro-optic performance of a multidomain MD IPS-LCD with chevron electrode is simulated using 3-D FEM method. Results indicate that the multi-domain structure greatly widens the viewing angle, shortens the response time, and suppresses the angular-dependent VT variation and color shift. These advantages are particularly attractive for the high quality LCD TV and computer monitor applications.

\section{REFERENCES}

[1] S. T. Wu and D. K. Yang, Reflective Liquid Crystal Displays. Hoboken, NJ: Wiley, 2001.

[2] A. Takeda, S. Kataoka, T. Sasaki, H. Chida, H. Tsuda, K. Ohmuro, T. Sasabayashi, Y. Koike, and K. Okamoto, "A super-high image quality multi-domain vertical alignment LCD by new rubbing-less technology," in SID'98 Dig., 1998, pp. 1077-1080.

[3] Y. Koike and K. Okamoto, "Super high quality MVA-TFT liquid crystal displays," Fujitsu Sci. Tech. J., vol. 35, pp. 221-228, Dec. 1999.
[4] H. Kim, J. Song, S. Park, J. Lyu, J. Souk, and K. Lee, "PVA technology for high performance LCD monitors," J. SID, vol. 1, pp. 3-8, Dec. 2000.

[5] S. Kim, "The world's largest (82-in.) TFT LCD," in SID'05 Dig., 2005 , pp. $1842-1847$.

[6] Y. Ishii, S. Mizushima, and M. Hijikigawa, "High performance TFTLCD's for AVC applications," in SID Symp. Dig., 2001, p. 1090.

[7] Y. Yamada, K. Miyachi, M. Kubo, S. Mizushima, Y. Ishii, and M. Hijikigawa, "Fast response and wide viewing angle technologies for LC-TV application," in Proc. Int. Display Workshop, 2002, p. 203.

[8] R. Kiefer, B. Webber, F. Windscheid, and G. Baur, "In-plane switching of nematic liquid crystals," Jpn. Displays, p. 547, 1992.

[9] M. Oh-e and K. Kondo, "Electro-optical characteristics and switching behavior of the in-plane switching mode," Appl. Phys. Lett., vol. 67, pp. 3895-3897, Dec. 1995.

[10] M. Oh-e, M. Yoneya, and K. Kondo, "Switching of negative and positive dielectro-anisotropic liquid crystals by the in-plane electric field," $J$. Appl. Phys., vol. 82, pp. 528-535, Jul 1997.

[11] S. Aratani, H. Klausmann, M. Oh-e, M. Ohta, K. Ashizawa, K. Yanagawa, and K. Kondo, "Complete suppression of color shift in in-plane switching mode liquid crystal displays with a multidomain structure obtained by unidirectional rubbing," Jpn. J. Appl. Phys., vol. 36, pp. L27-L29, Jan. 1997. 
[12] Y. Mishima, T. Nakayama, N. Suzuki, M. Ohta, S. Endoh, Y. Iwakabe, and H. Kagawa, "Development of a 19-in.-diagonal UXGA super TFT-LCM applied with Super-IPS technology," in SID Symp. Dig., 2000, pp. 260-263.

[13] S. T. Wu, "Nematic liquid crystal modulator with response time less than $100 \mu \mathrm{s}$ at room temperature," Appl. Phys. Lett., vol. 57, pp. 986-988, Sep. 1990.

[14] C. Xiang, X. Sun, and X. Yin, "Fast response wide viewing angle liquid crystal cell with double-side fringe-field switching," Appl. Phys. Lett., vol. 83, pp. 5154-5156, Dec. 2003.

[15] C. Xiang and X. Sun, "Electro-optical properties of a nematic liquid crystal cell by double-side fringe-field switching," J. Appl. Phys., vol. 96, pp. 3520-3523, Sep. 2004.

[16] Y. Sun, Z. Zhang, H. Ma, X. Zhu, and S. T. Wu, "Optimal rubbing angle for reflective in-plane switching liquid crystal displays," Appl. Phys. Lett., vol. 81, pp. 4907-4909, Dec. 2002.

[17] J. Chen, K. Kim, J. Jyu, J. Souk, J. Kelly, and P. Bos, "Optimum film compensation modes for TN and VA LCDs," in SID Symp. Dig., 1998, pp. 315-318.

[18] Y. Saitoh, S. Kimura, K. Kusafuka, and H. Shimizu, "Optimum film compensation of viewing angle of contrast in in-plane-switching-mode liquid crystal display," Jpn. J. Appl. Phys., vol. 37, pp. 4822-4828, Sep. 1998.

[19] T. X. Wu, R. Lu, Q. Hong, Y. Huang, X. Zhu, Z. Ge, and S. T. Wu, “On the modeling of liquid crystal displays," in Proc. 16th Annu. Meeting of IEEE Lasers \& Electro-Optics Soc., 2003, p. 886.

[20] P. G. de Gennes and J. Prost, The Physics of Liquid Crystals, 2nd ed. New York: Oxford Univ. Press, 1993.

[21] G. Dhatt and G. Touzoy, The Finite Element Method Displayed. New Delhi, India: Thomson, 1984.

[22] Y. W. Kwon, The Finite Element Method Using MATLAB. Boca Raton, FL: CRC, 1997.

[23] J. E. Anderson, P. E. Watson, and P. J. Bos, LC3D: Liquid Crystal Display 3-D Director Simulator Software and Technology Guide. Boston, MA: Artech House, 1993.

[24] A. Lien, "Extended Jones matrix representation for twisted nematic liquid-crystal display at oblique incidence," Appl. Phys. Lett., vol. 57, pp. 2767-2769, Dec. 1990.

[25] A. Lien, "A detailed derivation of extended Jones matrix representation for twisted nematic liquid crystal displays," Liq. Cryst., vol. 22, pp. 171-175, Feb. 1997.

[26] F. D. Pasqual, H. Deng, F. A. Fernandez, S. E. Day, J. B. Davies, M. Johnson, A. A. Put, J. M. Eerenbeemd, J. Haaren, and J. Chapman, "Theoretical and experimental study of nematic liquid crystal display cells using the in-plane-switching mode," IEEE Trans. Electron Devices, vol. 46, no. 4, pp. 661-667, Apr. 1999.

[27] S. H. Lee, S. L. Lee, and H. Kim, "Electro-optic characteristics and switching principle of a nematic liquid crystal cell controlled by fringefield switching," Appl. Phys. Lett., vol. 73, pp. 2881-2883, Nov. 1998.

[28] I. W. Wu, D. L. Ting, and C. Chang, "Advancement in wide viewing angle LCDs,” 6th Int. Display Workshops, pp. 383-386, 1999.

[29] H. Klausmann, S. Aratani, and K. Kondo, "Optical characterization of the in-plane switching effect utilizing multidomain structures," J. Appl. Phys., vol. 83, pp. 1854-1886, Feb.? 1998.

[30] S. Jung, S. Jang, K. Park, S. Lee, C. Seo, and W. Park, "Electro-optical analysis of super-IPS LCD's by using 3-D molecular director simulation of TFT-LCDs," in SID Symp. Dig., vol. 35, 2004, p. 618.

[31] S. T. Wu, "Phase-matched biaxial compensation film for LCD's," SID Symp. Dig., vol. 26, p. 555, 1995.

[32] I. C. Khoo and S. T. Wu, Optics and Nonlinear Optics of Liquid Crystals, Singapore: World Scientific, 1993.

[33] R. Lu, X. Zhu, S. T. Wu, Q. Hong, and T. X. Wu, "Ultra wide view liquid crystal displays," J. Display Tech., pp. 3-14, Sep. 2005.

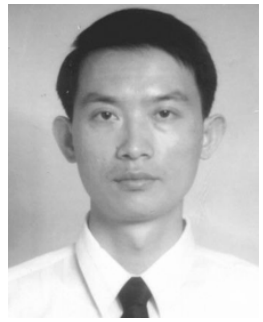

Ruibo Lu received the M.S. degree in applied physics from Department of Physics, East China University of Science and Technology, Shanghai, China, in 1995, and the Ph.D. degree in optics from Department of Physics, Fudan University, Shanghai, China, in 1998. His research work for the Ph.D. degree focused on liquid crystal alignment and ferroelectric liquid crystal devices for display and advanced optical applications.

He was part of the faculty in Department of Physics, and later in Department of Optical Science and Engineering, Fudan University, Shanghai, China, from 1998 to 2001. He was an optical engineer in Lightwaves 2020 Inc., San Jose, CA, from 2001 to 2002. Since then, he joined the School of Optics/CREOL (now as College of Optics and Photonics), University of Central Florida, Orlando, as a research scientist. His research interests include liquid crystal display technology, wide viewing angle for liquid crystal TVs, liquid crystal components for optical communications and optical imaging using liquid crystal medium.

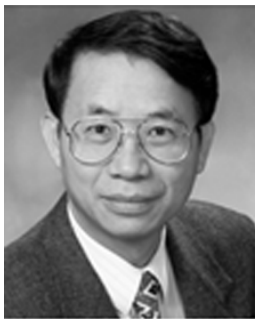

Shin-Tson Wu (M'98-SM'99-F'04) received the B.S. degree in physics from National Taiwan University, and the Ph.D. degree from the University of Southern California, Los Angeles.

$\mathrm{He}$ is currently a PREP professor at College of Optics and Photonics, University of Central Florida (UCF), Orlando. Prior to joining UCF in 2001, he worked at Hughes Research Laboratories, Malibu, CA, for 18 years. His studies at UCF concentrate in foveated imaging, bio-photonics, optical communications, liquid crystal displays, and liquid crystal materials. He has coauthored 2 books, Reflective Liquid Crystal Displays (Wiley, 2001) and Optics and Nonlinear Optics of Liquid Crystals (World Scientific, 1993), four book chapters, and over 220 journal papers.

Dr. Wu is a Fellow of the Society for Information Display (SID) and Optical Society of America (OSA).

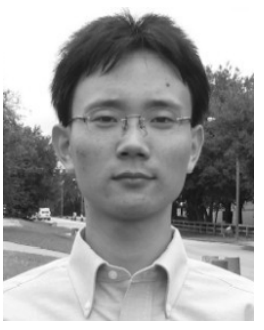

Zhibing Ge received the B.S. and M.S. degrees in electrical engineering from Zhejiang University, Hangzhou, China, and University of Central Florida, Orlando, in 2002 and 2004, respectively, and is currently working toward the Ph.D. degree at Department of Electrical and Computer Engineering, University of Central Florida, Orlando. His Ph.D. study concentration is in liquid crystal display modeling, transflective liquid crystal displays, and numerical analyses and optimization of liquid crystal devices.

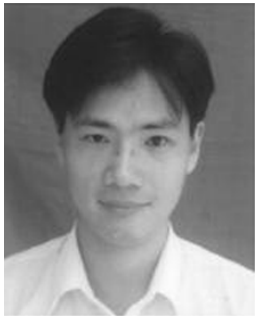

Qi Hong received B.S. degree from the Nanjing University of Aeronautics and Astronautics, Nanjing, China, in 1992, and the M.S.E.E. degree from the University of Central Florida, Orlando, in 2002, where he is currently working toward the Ph.D. degree in the electrical engineering. His doctoral research topics include liquid crystal device modeling, wide viewing angle and fast response liquid crystal display.

He was design engineer at the Xiaxin Electronics Company Ltd., Xiamen, China, from 1992 to 2000.

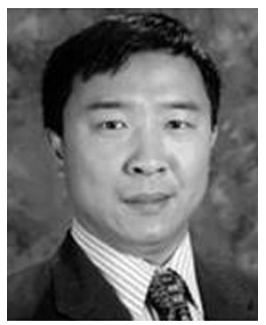

Thomas X. Wu received the B.S.E.E. and M.S.E.E degrees from the University of Science and Technology of China (USTC), Anhui, China, in 1988 and 1991, respectively, and the M.S. and Ph.D. degrees in electrical engineering from the University of Pennsylvania, Philadelphia, in 1997 and 1999, respectively.

From 1991 to 1995 , he was with the faculty of the Department of Electrical Engineering and Information Science, USTC, as an Assistant and Lecturer. In Fall 1999, he joined the Department of Electrical and Computer Engineering, University of Central Florida (UCF), Orlando, as an Assistant Professor. His current research interests and projects include complex media, liquid crystal devices, electronic packaging of RF SAW devices, electrical machinery, magnetics and EMC/EMI in power electronics, chaotic electromagnetics, millimeter-wave circuits, and CMOS/BiCMOS RFICs.

Dr. Wu was awarded the Distinguished Researcher Award from the College of Engineering and Computer Science, University of Central Florida, in April 2004. Recently, he was listed in Who's Who in Science and Engineering, Who's Who in America, and Who's Who in the World. 